\title{
Article \\ Predictive Value of the Phase Angle for Analgesic Efficacy in Lumbosacral Transforaminal Block
}

\author{
Jeayoun Kim ${ }^{1,+}$, Hue Jung Park ${ }^{2,+} \mathbb{D}$, Woo Seog Sim ${ }^{1}$, Seungwon Lee ${ }^{1}$, Keoungah Kim ${ }^{1}$, Woo Jin Kim ${ }^{1}$ \\ and Jin Young Lee ${ }^{1, *(\mathbb{D}}$ \\ 1 Samsung Medical Center, Department of Anesthesiology and Pain Medicine, School of Medicine, \\ Sungkyunkwan University, Seoul 06351, Korea; jeayoun.kim@samsung.com (J.K.); \\ wooseog.sim@samsung.com (W.S.S.); seungwon0209.lee@samsung.com (S.L.); \\ keoungah.kim@samsung.com (K.K.); wj888.kim@samsung.com (W.J.K.) \\ 2 Department of Anesthesiology and Pain Medicine, Seoul St. Mary's Hospital, College of Medicine, \\ The Catholic University of Korea, Seoul 06591, Korea; huejung@catholic.ac.kr \\ * Correspondence: L7035@hanmail.net; Tel.: +82-2-3410-1929; Fax: +82-2-3410-6626 \\ + These authors contributed equally to this work.
}

Citation: Kim, J.; Park, H.J.; Sim, W.S.; Lee, S.; Kim, K.; Kim, W.J.; Lee, J.Y. Predictive Value of the Phase Angle for Analgesic Efficacy in Lumbosacral Transforaminal Block. J. Clin. Med. 2021, 10, 240. https:// doi.org/10.3390/jcm10020240

Received: 3 December 2020 Accepted: 8 January 2021 Published: 12 January 2021

Publisher's Note: MDPI stays neutral with regard to jurisdictional clai$\mathrm{ms}$ in published maps and institutional affiliations.

Copyright: (C) 2021 by the authors. Licensee MDPI, Basel, Switzerland. This article is an open access article distributed under the terms and conditions of the Creative Commons Attribution (CC BY) license (https:// creativecommons.org/licenses/by/ $4.0 /)$.

\begin{abstract}
The mechanism of low back and leg pain involves mixed neuropathic and nociceptive components. Spinal neuropathic pain is related to increased levels of inflammatory cytokines and disrupted and increased permeability of the blood-spinal cord barrier, originally composed of tight junctions of capillary endothelial cells surrounded by lamina. The phase angle (PA) estimates cell membrane integrity using bioelectrical impedance analysis. We evaluated the predictive value of the PA for analgesic efficacy in lumbosacral transforaminal block. We retrospectively collected data from 120 patients receiving transforaminal blocks for lumbosacral radicular pain and assessed the PA before and $5 \mathrm{~min}$ following the block. Responders (group R) and non-responders (group N) were defined by $\geq 50 \%$ and $<50 \%$ pain reduction, respectively, on the numerical rating scale, $30 \mathrm{~min}$ following the block; clinical data and the PA were compared. Among the 109 included patients, $50(45.9 \%)$ and $59(54.1 \%)$ had $\geq 50 \%$ and $<50 \%$ pain reduction, respectively. In group $\mathrm{N}$, the PA change ratio showed $88.1 \%$ specificity, $32.0 \%$ sensitivity, and $62.4 \%$ accuracy; a ratio of $<0.087$ at $5 \mathrm{~min}$ following the block predicted non-response. A PA change ratio of $<0.087$ at 5 min following lumbar transforaminal blocks predicted non-responders with high specificity.
\end{abstract}

Keywords: lumbosacral; pain; phase angle; radicular; transforaminal block

\section{Introduction}

Lumbar epidural injection of local anesthetics and steroids is one of the methods of managing low back and radicular pain [1]. As one of several approaches, the transforaminal approach can deliver a small volume of injectate close to the site of pathology, presumably into a nerve root, to reduce inflammation and swelling [1-5]. However, objective metrics for assessing pain relief have not been well evaluated. Commonly used self-reported pain questionnaires are based on patient subjective responses, which may be affected by various factors, such as comorbid conditions and/or psychosocial causes [6-8]. Several objective pain tools, including skin conductance, nociception index, and pupillometry, have been reported; however, they involve high costs, limited availability, invasiveness, and/or various levels of accuracy [9]. In our pervious study on lumbar transforaminal blocks, we evaluated the correlation between perfusion index change and analgesic efficacy. We calculated the perfusion index change ratio from the perfusion index values before and 5 min after the block, and a ratio of $>0.27$ was found to be significantly related to pain relief; however, the perfusion index value was greatly changed by the assessing environment [10]. Early identification of the pain status is crucial for developing further treatment plans [6]. In cases where pain persists after the block, physicians consider further work-up to clearly diagnose 
and/or repeat blocks to relieve symptoms. Therefore, pain assessment after treatment is a cornerstone for decision-making regarding subsequent management. Pain monitoring by combining multiple autonomic signals (finger photoplethysmogram amplitude, skin conductance, heart rate, and heart rate variability) has shown limited correlation for detecting nociception [11]. Bioelectrical impedance analysis (BIA) is a technique used to measure body impedance by introducing a small current to the body; it is based on the principle that biological tissues act as conductors, semiconductors, or insulators [12,13]. The phase angle (PA) is a value derived from BIA [14]. The PA is estimated based on the ratio of reactance to resistance [12]. Resistance is the degree of obstruction of current flow caused by non-conductive tissue components, such as fat mass [12]. Reactance is an additional conduction delay, which is related to the capacitance of the cell membrane and tissue interfaces [12]. Reactance involves the integrity of cell membranes and the body cell mass [12]. The PA is positively associated with reactance and inversely associated with resistance [12]. In previous studies, a low PA has been observed in cases of a disrupted cellular membrane (malnutrition and inflammation) $[15,16]$. The PA may also predict poor survival outcomes in colorectal, lung, breast, or pancreatic cancers, leukemia, human immunodeficiency infection, and end-stage renal disease $[17,18]$. Recently, the PA showed diagnostic value in dysmobility syndromes characterized by impaired muscle function and performance status [12]. Lumbosacral radicular pain has mixed characteristics of neuropathic and nociceptive pain by spinal nerve roots inflammation and impingement [19-21]. We attempted to determine whether PA could reflect the pain status by measuring cell integrity before and after neural inflammation treatment. This study was performed to evaluate the predictive value of PA for analgesic efficacy in patients undergoing lumbosacral transforaminal blocks.

\section{Materials and Methods}

\subsection{Patients}

We retrospectively reviewed the electronic medical records of patients with lower back and leg pain, who underwent a transforaminal block between February and September 2020 at a single tertiary care hospital; the patients were aged between 46 and 84 years. The inclusion criteria were as follows: (a) a primary diagnosis of lower back pain radiating to the lower limbs, (b) availability of a cross-sectional imaging study (either CT (computed tomography) or MRI (magnetic resonance imaging)) of the lumbosacral spine in patients diagnosed with spinal stenosis or herniated nucleus pulposus. This study was approved by our departmental ethics committee (SMC 2020-09-170) and registered with CRIS (Clinical Research Information Service of the Korea National Institute of Health, http:/ / cris.nih. go.kr/cris/index.jsp, KCT0005642). The need for individual consent was waived by the institutional review board, as this was a retrospective study involving medical record review. Among the 120 patients assessed for eligibility, 11 were excluded due to zero PA values at T0 or T5, which led to zero PA change ratios on calculation; thus, the data of 109 patients were analyzed. We defined responders, or group $\mathrm{R}$, as patients who showed a reduction of $\geq 50 \%$ on the numerical rating scale (NRS, ranging from $0=$ no pain to $10=$ absolutely intolerable pain) for pain at T30, and non-responders, or group N, as those who showed a reduction of less than $50 \%$ at T30 (Figure 1). The demographic and clinical data are summarized in Table 1. 


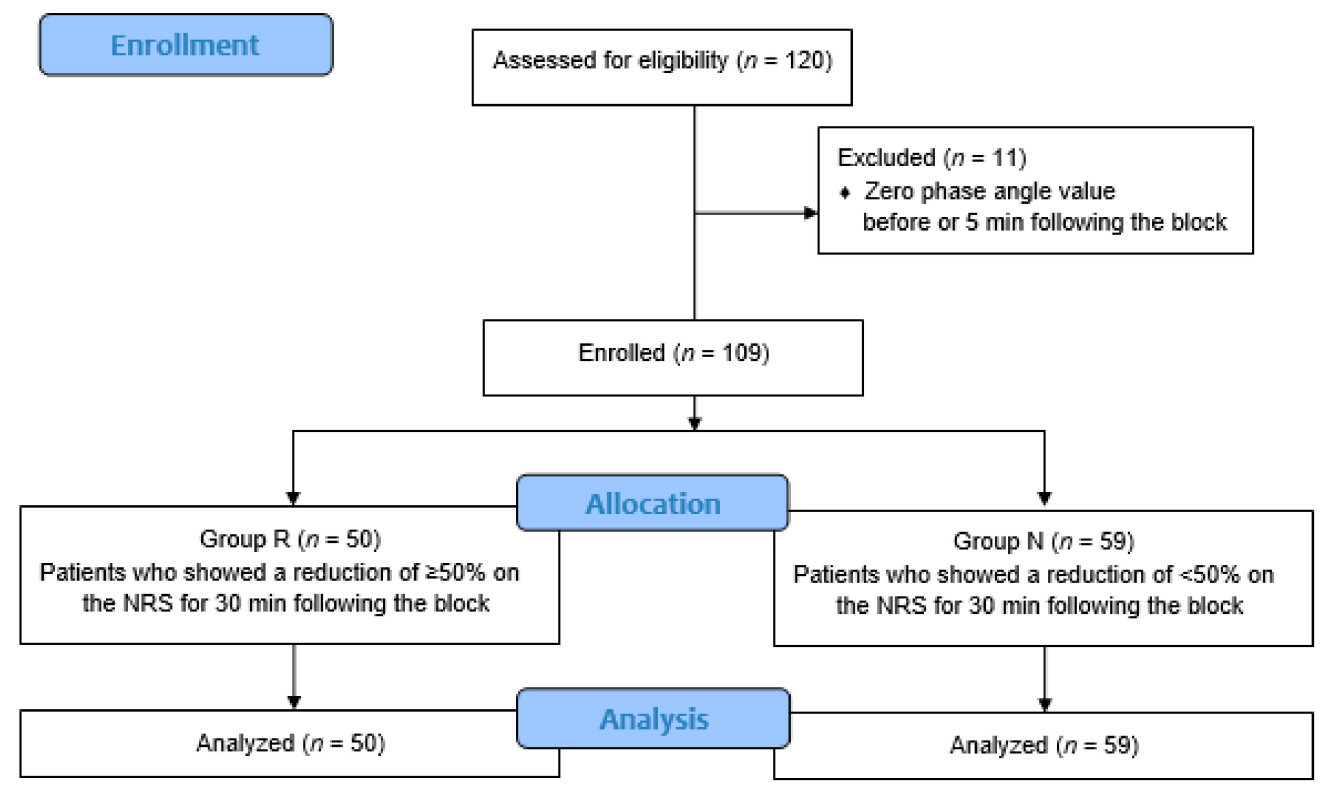

Figure 1. Flow diagram of the study. NRS: numerical rating scale.

Table 1. Demographic and clinical characteristics.

\begin{tabular}{|c|c|c|c|c|}
\hline Title & $\begin{array}{l}\text { All Patients } \\
\quad(n=109)\end{array}$ & $\begin{array}{c}\text { Group R } \\
(n=50)\end{array}$ & $\begin{array}{c}\text { Group N } \\
(n=59)\end{array}$ & $p$-Value \\
\hline Age (year) & $69.3 \pm 8.0$ & $69.2 \pm 8.1$ & $69.4 \pm 7.9$ & 0.895 \\
\hline Sex (male/female) & $65 / 44$ & $31 / 19$ & $34 / 25$ & 0.643 \\
\hline Body mass index $\left(\mathrm{kg} / \mathrm{m}^{2}\right)$ & $25.8 \pm 3.2$ & $26.7 \pm 3.5$ & $25.1 \pm 2.8$ & 0.014 \\
\hline Diagnosis & & & & 1.000 \\
\hline Spinal stenosis & $98(89.9 \%)$ & $45(90.0 \%)$ & $53(89.8 \%)$ & \\
\hline HNP & $11(10.1 \%)$ & $5(10.0 \%)$ & $6(10.2 \%)$ & \\
\hline Duration of pain (month) & & & & 0.340 \\
\hline$<3$ & $4(3.7 \%)$ & $3(6.0 \%)$ & $1(1.7 \%)$ & \\
\hline $3-12$ & $21(19.3 \%)$ & $11(22.0 \%)$ & $10(16.9 \%)$ & \\
\hline$>12$ & $84(77.1 \%)$ & $36(72.0 \%)$ & $48(81.4 \%)$ & \\
\hline Lesion level & & & & 0.639 \\
\hline L2-3 & $1(0.9 \%)$ & $1(2.0 \%)$ & $0(0.0 \%)$ & \\
\hline L3-4 & $23(21.1 \%)$ & $11(22.0 \%)$ & $12(20.3 \%)$ & \\
\hline L4-5 & $64(58.7 \%)$ & $27(54.0 \%)$ & $37(62.7 \%)$ & \\
\hline L5-S1 & $21(19.3 \%)$ & $11(22.0 \%)$ & $10(17.0 \%)$ & \\
\hline Lesion severity & & & & 0.038 \\
\hline Mild & $0(0.0 \%)$ & $0(0.0 \%)$ & $0(0.0 \%)$ & \\
\hline Moderate & $66(60.6 \%)$ & $25(50.0 \%)$ & $41(69.5 \%)$ & \\
\hline Severe & $43(39.4 \%)$ & $25(50.0 \%)$ & $18(30.5 \%)$ & \\
\hline Injection level & & & & 0.181 \\
\hline L2 & $1(0.9 \%)$ & $0(0.0 \%)$ & $1(1.7 \%)$ & \\
\hline L3 & $16(14.7 \%)$ & $10(20.0 \%)$ & $6(10.2 \%)$ & \\
\hline $\mathrm{L} 4$ & $47(43.1 \%)$ & $17(34.0 \%)$ & $30(50.8 \%)$ & \\
\hline L5 & $41(37.6 \%)$ & $20(40.0 \%)$ & $21(35.6 \%)$ & \\
\hline $\mathrm{S} 1$ & $4(3.7 \%)$ & $3(6.0 \%)$ & $1(1.7 \%)$ & \\
\hline Injection side & & & & 0.301 \\
\hline Left/Right & $56 / 53$ & $23 / 27$ & $33 / 26$ & \\
\hline Attempt number & & & & 0.248 \\
\hline $1 / 2$ & $106 / 3$ & $50 / 0$ & $56 / 3$ & \\
\hline
\end{tabular}

All data are presented as the mean $\pm S D$ or number (\%) of patients. HNP: herniated nucleus pulposus; Group R: patients who showed a reduction of $\geq 50 \%$ on the numerical rating scale for pain $30 \mathrm{~min}$ following the block; Group N: patients who showed a reduction less than $50 \% ; p$-value $<0.05$ was considered statistically significant. 


\subsection{Intervention}

All procedures were performed under fluoroscopic guidance and were standardized. The lesion level for transforaminal injections was selected based on clinical manifestations, physical examination, and review of imaging studies. Lesion severity was categorized at any of three different levels (mild, moderate, or severe) by reviewing the imaging data. Exclusion criteria included any history of lumbosacral surgery, lumbosacral neuroplasty, neoplastic disease, or peripheral vascular disease. Patients were placed in the prone position, and anteroposterior and lateral view images were obtained using a C-arm (OEC series 9800, General Electronics, New York, NY, USA) to ensure proper site of entry. Following aseptic preparation and application of $1 \%$ lidocaine, a 23-gauge Tuohy needle (Tae-Chang Industrial Co., Seoul, Korea) was inserted into the skin surface over the upper quadrant of the target foramen. Aspirations were routinely performed for assessing the presence of blood or cerebrospinal fluid. When negative for aspirate, $0.5-2 \mathrm{~mL}$ of contrast medium (Omnipaque $^{\circledR}, 300 \mathrm{mgI} \cdot \mathrm{mL}^{-1}$, GE Healthcare, Little Chalfont, Buckinghamshire, UK) was injected to confirm that the point was well placed in the epidural space. After confirming that the contrast had spread throughout the epidural space, a total volume of $5 \mathrm{~mL}$ (containing $0.4 \%$ lidocaine, dexamethasone, hyaluronidase $750 \mathrm{IU}$, and normal saline) was infused. Following the procedure, patients were observed for any adverse effects. Whole-body BIA measurement was assessed using the Inbody S10 (Biospace Co., Ltd., Seoul, Korea). Following the standardized protocol, electrodes were placed on the bilateral hands and bare feet for $2 \mathrm{~min}$ [12] (Figure 2). The PA was calculated using the reactance and resistance database obtained at $50 \mathrm{~Hz}$; we collected the PA value of the affected lower limb [12] prior to treatment (T0) and $5 \mathrm{~min}$ following transforaminal injection (T5). Temperature was assessed using a touch thermometer (IntelliVue MP70 patient monitor, Philips Healthcare, Best, the Netherlands) on the dorsum of the foot of the affected lower limb at T0 and T5. The room temperature was maintained at $23-25^{\circ} \mathrm{C}$. Pain and cold sensations of the affected lower limb were scored and recorded using the NRS at T0 and 30 min following transforaminal injection (T30).

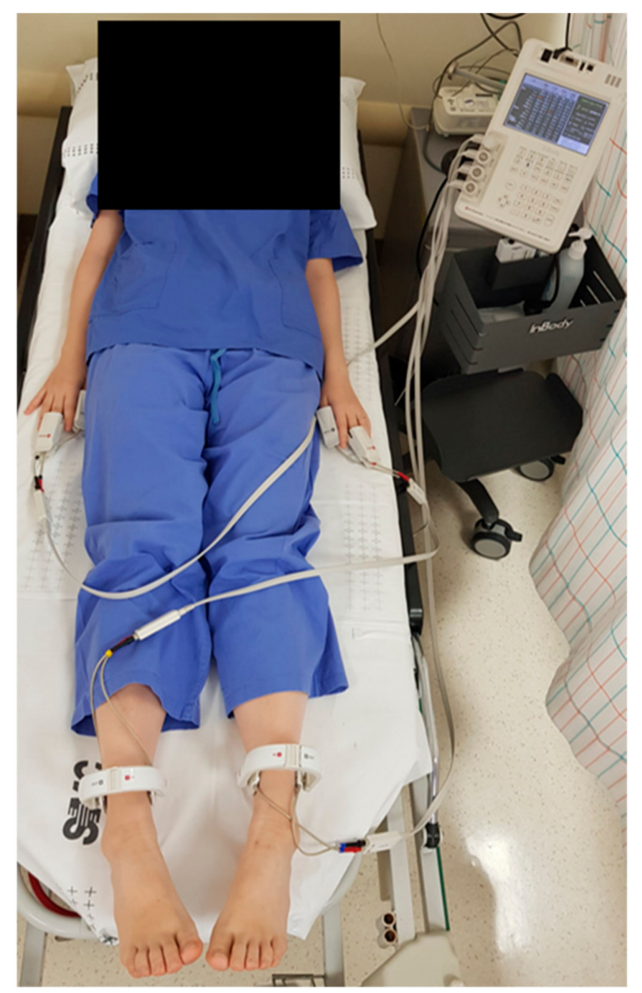

Figure 2. Whole-body bioelectrical impedance analysis measurement. 


\subsection{Statistical Analysis}

All data were analyzed using SAS 9.4 (SAS Institute, Cary, NC, USA). Data are expressed as the mean \pm standard deviation $(S D)$ or numbers (proportion), as appropriate. Demographic data for the two groups were compared using a Chi-square test, $t$-test, or Fisher's exact test. To minimize individual variance in PA absolute values, we calculated the PA change ratio (PA at $\mathrm{T} 5-\mathrm{PA}$ at $\mathrm{T} 0 / \mathrm{PA}$ at $\mathrm{T} 0$ ). Temperature change (temperature at T5 - temperature at T0) was calculated. The PA change ratio was compared using the Wilcoxon rank sum test. The cut-off value of the PA change ratio was analyzed using Youden's index. A $p$-value less than 0.05 was considered statistically significant.

\section{Results}

Age, sex, diagnosis, duration of pain, lesion level, injection level, injection side, and attempt number did not differ between the two groups (Table 1). Body mass index and lesion severity was different between the groups $(p=0.014, p=0.038$, respectively) (Table 1$)$. The PA value, PA change, PA change ratio, and temperature change are presented in Table 2. The PA value was higher at T0 and T5 in group R, but no significant difference was observed between the groups (Table 2). PA change, PA change ratio, and temperature change were not different between the groups (Table 2). In group N, the cut-off value of the PA change ratio was $<0.087$ at $\mathrm{T} 5$; the area under the receiver operating characteristic curve was 0.521 . The PA change ratio showed $32.0 \%$ sensitivity, $88.1 \%$ specificity, $62.4 \%$ accuracy, and Youden's index of 0.201 (Table 3). Pain severity was different at T30 between the groups $(p<0.001)$ (Table 4). Cold sensation did not differ at T0 and T30 between the groups (Table 4). None of the cases showed any evidence of dural puncture or neurologic complications.

Table 2. Phase angle and temperature.

\begin{tabular}{ccccc}
\hline & $\begin{array}{c}\text { All Patients } \\
(\boldsymbol{n}=\mathbf{1 0 9 )}\end{array}$ & $\begin{array}{c}\text { Group R } \\
(\boldsymbol{n}=\mathbf{5 0 )}\end{array}$ & $\begin{array}{c}\text { Group N } \\
(\boldsymbol{n}=\mathbf{5 9 )})\end{array}$ & $\boldsymbol{p}$-Value \\
\hline Phase angle value & & & & \\
T0 & $6.42 \pm 8.3$ & $7.63 \pm 12.0$ & $5.40 \pm 1.5$ & 0.944 \\
T5 & $6.64 \pm 9.2$ & $8.09 \pm 13.4$ & $5.42 \pm 1.6$ & 0.733 \\
\hline Phase angle change & $0.22 \pm 1.8$ & $0.46 \pm 2.3$ & $0.02 \pm 1.3$ & 0.594 \\
Phase angle change ratio & $0.05 \pm 0.4$ & $0.09 \pm 0.6$ & $0.02 \pm 0.3$ & 0.713 \\
Temperature change & $-0.08 \pm 0.2$ & $-0.08 \pm 0.2$ & $-0.07 \pm 0.2$ & 0.933 \\
\hline
\end{tabular}

All data are presented as the mean $\pm S D$. T0: before treatment; T5: 5 min following the block; Phase angle change (phase angle at T5 phase angle at T0); Phase angle change ratio (phase angle at T5 - phase angle at T0/phase angle at T0); Temperature change (temperature at T5 - temperature at T0); Group R: patients who showed a reduction of $\geq 50 \%$ on the numerical rating scale for pain 30 min following the block; Group N: patients who showed a reduction less than $50 \%$; $p$-value $<0.05$ was considered statistically significant.

Table 3. Diagnostic assessment of the phase angle change ratio in non-responders.

\begin{tabular}{cc}
\hline & Group N $(\boldsymbol{n}=\mathbf{5 9})$ \\
\hline AUROC & $0.521(95 \%$ CI $0.408-0.633)$ \\
Cut-off value & 0.087 \\
Sensitivity & $32.0 \%(95 \%$ CI $0.208-0.458)$ \\
Specificity & $88.1 \%(95 \%$ CI $0.775-0.941)$ \\
Accuracy & $62.4 \%(95 \%$ CI $0.530-0.709)$ \\
Youden's index & 0.201
\end{tabular}

AUROC: area under the receiver operating characteristic curve; CI: confidence interval; Group N: patients who showed a reduction less than $50 \%$. 
Table 4. Pain severity and cold sensation over time.

\begin{tabular}{ccccc}
\hline & $\begin{array}{c}\text { All Patients } \\
(\boldsymbol{n}=\mathbf{1 0 9 )}\end{array}$ & $\begin{array}{c}\text { Group R } \\
(\boldsymbol{n}=\mathbf{5 0 )}\end{array}$ & $\begin{array}{c}\text { Group N } \\
(\boldsymbol{n}=\mathbf{5 9 )}\end{array}$ & $\boldsymbol{p}$-Value \\
\hline Pain severity (NRS) & & & & \\
T0 & $6.48 \pm 2.0$ & $6.72 \pm 2.2$ & $6.27 \pm 1.9$ & 0.346 \\
T30 & $3.66 \pm 2.4$ & $1.70 \pm 1.7$ & $5.32 \pm 1.4$ & $<0.001$ \\
\hline Cold sensation (NRS) & & & \\
T0 & $2.06 \pm 3.0$ & $1.66 \pm 3.0$ & $2.39 \pm 3.0$ & 0.122 \\
T30 & $1.26 \pm 2.2$ & $0.92 \pm 2.0$ & $1.54 \pm 2.3$ & 0.094 \\
\hline
\end{tabular}

All data are presented as the mean $\pm S D$. T0: before treatment; T30: 30 min following the block; NRS: numerical rating scale; Group R: patients who showed a reduction of $\geq 50 \%$ on the numerical rating scale for pain $30 \mathrm{~min}$ following the block; Group N: patients who showed a reduction less than $50 \%$; $p$-value $<0.05$ was considered statistically significant.

\section{Discussion}

In the present study, we found that a PA change ratio of $<0.087$ showed high specificity for identifying non-responders following transforaminal block. We speculate that low PA change ratios are attributable to lesser relief of inflammation and/or swelling of the affected nerve roots and are related to non-improvement of cellularity and non-response after epidural injections. Excessive nerve root swelling is a known risk factor for persistent or recurrent radiating pain after lumbar surgery [22]. It leads to nerve root compression with hyperalgesia, allodynia, and radiating pain [22]. Therefore, evaluation of non-responders after blocks is critical for deciding on further treatment. PA can be helpful as a simple, noninvasive, and easy-to-use pain tool when used along with a subjective pain questionnaire.

Lumbosacral radicular pain is caused by irritation or compression of the affected nerve root [23]. It is caused by chemically mediated inflammatory reactions, as well as by a direct mass effect on the nerve root [24]. The mechanism of low back and leg pain involves mixed components of neuropathic and nociceptive mechanisms [25]. Spinal neuropathic pain is related to increased levels of inflammatory cytokines and disrupted and increased permeability of the blood-spinal barrier, originally composed of tight junctions of capillary endothelial cell surrounded by lamina [26]. Dysfunction and breakage of the blood-spinal cord barrier is observed in several neurodegenerative disorders, peripheral neural injury-induced inflammatory pain, and chemotherapy-induced neuropathic pain $[26,27]$. In a rat sciatic nerve model, recruitment of inflammatory materials outside the nerve led to damage of the epineurium and depletion of epineural adipocytes [21,28]. Simultaneously, pro-nociceptive and pro-inflammatory mediators can enter the sciatic nerve through microlesions caused by neural damage [28]. Therefore, non-myelinating Schwann cells may no longer protect non-myelinated neurons, causing tactile allodynia and loss of function of thermal sensitivity [28]. Transforaminal block is a valid procedure for the diagnosis and treatment of lumbosacral radicular pain, and acts by reducing inflammation and edema [29]. Steroid and local anesthetics through the foramen dilute inflammatory cytokines, reduce adhesion, improve blood circulation, suppress affected neural ectopic discharge, and reduce central sensitization [30]. We attempted to quantify pain changes using an objective tool after resolving nerve root inflammation with transforaminal block. Although the perfusion index was associated with pain relief following transforaminal block, perfusion index measurement is considerably sensitive to movement, and it fluctuates rapidly in response to surgical or other noxious stimuli, temperature, and stress, leading to changes in the perfusion index [31,32]. The PA is a parameter of BIA measurement, which reflects balance between cell hydration and body mass [33]. A low PA value is associated with cellular membrane damage and cell death, and a high PA value is correlated with proper cell membrane or cell function [33]. The PA depends on tissue features, cell size, cell function, and cellularity. Moreover, the PA is known as a cellular health indicator, but the prognostic value of PA may differ in various clinical situations [33,34]. 
In this study, we aimed to evaluate whether the PA has predictive value for analgesic efficacy after lumbosacral transforaminal blocks. There was no significant difference in the PA values between the groups. However, the PA change ratio showed $88.1 \%$ specificity in non-responders. The cut-off value of the PA change ratio was $<0.087$ at $5 \mathrm{~min}$ following the block. We followed up the PA at 5 min after the block because the perfusion index change ratio was significant at that time point, and it correlated with pain relief [10]. We suspect that the PA evaluation time was inadequate for reflecting improvement of inflammation and cellular integrity of the affected nerve roots after transforaminal blocks. Additionally, we included patients with different pain durations (acute and chronic pain), which may affect the functional dysmobility and inflammatory status, leading to bias regarding the PA value. Further studies will be needed to evaluate appropriate measurement times and to compare PA values between acute and chronic pain conditions. This study has several limitations. First, we categorized patients only using a numerical rating scale; we did not record the functional disability status. Second, the follow-up period of $30 \mathrm{~min}$ for detecting a response was inadequate for evaluating block efficacy. Third, we did not measure the nutrition status and pro-inflammatory or inflammatory markers in each patient; CRP (Creactive protein) and TNF- $\alpha$ (tumor necrosis factor) are known to be related with cell plasma membrane instability $[35,36]$. Fourth, we did not enroll a sham group to compare with the PA values of patients who did not receive blocks. Fifth, the sample was heterogeneous in terms of age, ranging from 46 to 84 years, and we did not perform age adjustment because there was no significant difference in age between the groups. Finally, the sample size was small, but the power was $82.9 \%$.

\section{Conclusions}

We observed that the PA change ratio has high specificity in identifying non-responders after lumbar transforaminal blocks. Further prospective controlled studies are needed to determine whether the PA provides superior diagnostic value in other forms of acute and chronic pain treatment.

Author Contributions: J.K.: investigation and writing; H.J.P. and W.S.S.: review; S.L., K.K., and W.J.K.: data curation and methodology; J.Y.L.: conceptualization, data curation, formal analysis, investigation, methodology, writing, review and editing. All authors have read and agreed to the published version of the manuscript.

Funding: This study was not supported by any external fund.

Institutional Review Board Statement: The study was conducted according to the guidelines of the Declaration of Helsinki, and approved by the Institutional Review Board of Samsung Medical Center (SMC 2020-09-170 on 8 October 2020).

Informed Consent Statement: Patient consent was waived due to retrospective study involving medical record review.

Data Availability Statement: The data presented in this study are available on request from the corresponding author. The data are not publicly available due to ethical reason.

Acknowledgments: The authors would like to thank to Samsung Medical Center biostatistics team for their statistical assistance and supervision.

Conflicts of Interest: The authors declare no conflict of interest.

\section{References}

1. Manchikanti, L.; Buenaventura, R.M.; Manchikanti, K.N.; Ruan, X.; Gupta, S.; Smith, H.S.; Christo, P.J.; Ward, S.P. Effectiveness of therapeutic lumbar transforaminal epidural steroid injections in managing lumbar spinal pain. Pain Physician 2012, 15, E199-E245. [PubMed]

2. Maus, T.P.; El-Yahchouchi, C.A.; Geske, J.R.; Carter, R.E.; Kaufmann, T.J.; Wald, J.T.; Diehn, F.E. Imaging Determinants of Clinical Effectiveness of Lumbar Transforaminal Epidural Steroid Injections. Pain Med. 2016, 17, 2176-2184. [CrossRef] [PubMed]

3. Rivera, C.E. Lumbar Epidural Steroid Injections. Phys. Med. Rehabil. Clin. N. Am. 2018, 29, 73-92. [CrossRef] [PubMed] 
4. Singh, J.R.; Cardozo, E.; Christolias, G.C. The Clinical Efficacy for Two-Level Transforaminal Epidural Steroid Injections. PMER 2017, 9, 377-382.

5. Gupta, R.; Singh, S.; Kaur, S.; Singh, K.; Aujla, K. Correlation between Epidurographic Contrast Flow Patterns and Clinical Effectiveness in Chronic Lumbar Discogenic Radicular Pain Treated with Epidural Steroid Injections Via Different Approaches. Korean J. Pain 2014, 27, 353-359. [CrossRef]

6. Pauli, J.; Starkweather, A.; Robins, J.L. Screening Tools to Predict the Development of Chronic Low Back Pain: An Integrative Review of the Literature. Pain Med. 2019, 20, 1651-1677. [CrossRef]

7. Hwang, S.; Choi, Y.J.; Jung, J.Y.; Choi, Y.; Ham, E.M.; Park, J.W.; Kwon, H.; Kim, D.K.; Kwak, Y.H. Pain Passport as a tool to improve analgesic use in children with suspected fractures in emergency departments. Korean J. Pain 2020, 33, 386-394. [CrossRef]

8. Cho, S.H.; Ko, S.H.; Lee, M.S.; Koo, B.S.; Lee, J.H.; Kim, S.H.; Chae, W.S.; Jin, H.C.; Lee, J.S.; Kim, Y.I. Development of the Geop-Pain questionnaire for multidisciplinary assessment of pain sensitivity. Korean J. Anesthesiol. 2016, 69, 492-505. [CrossRef]

9. Ledowski, T. Objective monitoring of nociception: A review of current commercial solutions. Br. J. Anaesth. 2019, 123, e312. [CrossRef]

10. Lee, J.Y.; Kim, E.D.; Kim, Y.N.; Kim, J.S.; Sim, W.S.; Lee, H.J.; Park, H.J.; Park, H.J. Correlation of Perfusion Index Change and Analgesic Efficacy in Transforaminal Block for Lumbosacral Radicular Pain. J. Clin. Med. 2019, 8, 51. [CrossRef]

11. Meijer, F.; Honing, M.; Roor, T.; Toet, S.; Calis, P.; Olofsen, E.; Martini, C.; van Velzen, M.; Aarts, L.; Niesters, M.; et al. Reduced postoperative pain using Nociception Level-guided fentanyl dosing during sevoflurane anaesthesia: A randomised controlled trial. Br. J. Anaesth. 2020, 125, 1070-1078. [CrossRef] [PubMed]

12. Jung, Y.W.; Hong, N.; Kim, C.O.; Kim, H.C.; Youm, Y.; Choi, J.; Rhee, Y. The diagnostic value of phase angle, an integrative bioelectrical marker, for identifying individuals with dysmobility syndrome: The Korean Urban-Rural Elderly study. Osteoporos. Int. 2020. [CrossRef] [PubMed]

13. Kushner, R.F. Bioelectrical impedance analysis: A review of principles and applications. J. Am. Coll. Nutr. 1992, 11, 199-209. [CrossRef] [PubMed]

14. Jensen, B.; Braun, W.; Both, M.; Gallagher, D.; Clark, P.; González, D.L.; Klückmann, K.; Bosy-Westphal, A. Configuration of bioelectrical impedance measurements affects results for phase angle. Med. Eng. Phys. 2020, 84, 10-15. [CrossRef] [PubMed]

15. Norman, K.; Stobäus, N.; Pirlich, M.; Bosy-Westphal, A. Bioelectrical phase angle and impedance vector analysis-Clinical relevance and applicability of impedance parameters. Clin. Nutr. 2012, 31, 854-861. [CrossRef]

16. Baumgartner, R.N.; Chumlea, W.C.; Roche, A.F. Bioelectric impedance phase angle and body composition. Am. J. Clin. Nutr. 1988, 48, 16-23. [CrossRef]

17. Yates, S.J.; Lyerly, S.; Manuel, M.; Tooze, J.A.; Klepin, H.D.; Powell, B.L.; Dralle, S.; Uprety, A.; Pardee, T.S. The prognostic value of standardized phase angle in adults with acute leukemia: A prospective study. Cancer Med. 2020, 9, 2403-2413. [CrossRef]

18. Hui, D.; Dev, R.; Pimental, L.; Park, M.; Cerana, M.A.; Liu, D.; Bruera, E. Association Between Multi-frequency Phase Angle and Survival in Patients With Advanced Cancer. J. Pain Symptom. Manag. 2017, 53, 571-577. [CrossRef]

19. Hasvik, E.; Schjølberg, T.; Jacobsen, D.P.; Haugen, A.J.; Grøvle, L.; Schistad, E.I.; Gjerstad, J. Up-regulation of circulating microRNA-17 is associated with lumbar radicular pain following disc herniation. Arthritis Res. Ther. 2019, 21, 186. [CrossRef]

20. Choi, Y.K. Lumbar foraminal neuropathy: An update on non-surgical management. Korean J. Pain 2019, 32, 147-159. [CrossRef]

21. Seol, T.K.; Lee, W.; Park, S.; Kim, K.N.; Kim, T.Y.; Oh, Y.N.; Jun, J.H. Effect of palmitoylethanolamide on inflammatory and neuropathic pain in rats. Korean J. Anesthesiol. 2017, 70, 561-566. [CrossRef] [PubMed]

22. Feng, Y.; Cai, F.; Chen, L.; Gu, Y.; Geng, D.C.; Yang, H.L.; Tang, T.S. Excessive swelling of nerve roots: Important factor for recurring sciatica after lumbar surgery. Orthopade 2020, 49, 502-509. [CrossRef] [PubMed]

23. Van Boxem, K.; Cheng, J.; Patijn, J.; van Kleef, M.; Lataster, A.; Mekhail, N.; Van Zundert, J. Lumbosacral radicular pain. Pain Pract. 2010, 10, 339-358. [CrossRef] [PubMed]

24. Valat, J.P.; Genevay, S.; Marty, M.; Rozenberg, S.; Koes, B. Sciatica. Best Pract. Res. Clin. Rheumatol. 2010, 24, 241-252. [CrossRef]

25. Attal, N.; Perrot, S.; Fermanian, J.; Bouhassira, D. The neuropathic components of chronic low back pain: A prospective multicenter study using the DN4 Questionnaire. J. Pain 2011, 12, 1080-1087. [CrossRef]

26. Wang, C.; Xu, K.; Wang, Y.; Mao, Y.; Huang, Y.; Liang, Y.; Liu, Y.; Hao, J.; Gu, X.; Ma, Z.; et al. Spinal cannabinoid receptor 2 activation reduces hypersensitivity associated with bone cancer pain and improves the integrity of the blood-spinal cord barrier. Reg. Anesth. Pain Med. 2020, 45, 783-791. [CrossRef]

27. Montague-Cardoso, K.; Pitcher, T.; Chisolm, K.; Salera, G.; Lindstrom, E.; Hewitt, E.; Solito, E.; Malcangio, M. Changes in vascular permeability in the spinal cord contribute to chemotherapy-induced neuropathic pain. Brain Behav. Immun. 2020, 83, 248-259. [CrossRef]

28. Cohnen, J.; Kornstädt, L.; Hahnefeld, L.; Ferreiros, N.; Pierre, S.; Koehl, U.; Deller, T.; Geisslinger, G.; Scholich, K. Tumors Provoke Inflammation and Perineural Microlesions at Adjacent Peripheral Nerves. Cells 2020, 9, 320. [CrossRef]

29. Van Helvoirt, H.; Apeldoorn, A.T.; Knol, D.L.; Arts, M.P.; Kamper, S.J.; van Tulder, M.W.; Ostelo, R.W. Transforaminal epidural steroid injections influence Mechanical Diagnosis and Therapy (MDT) pain response classification in candidates for lumbar herniated disc surgery. J. Back Musculoskelet. Rehabil. 2016, 29, 351-359. [CrossRef]

30. Fujiwara, A.; Watanabe, K.; Hashizume, K.; Shinohara, K.; Kawaguchi, M. Transforaminal vs Interlaminar Epidural Steroid Injection for Acute-Phase Shingles: A Randomized, Prospective Trial. Pain Physician 2018, 21, 373-382. [CrossRef] 
31. Mowafi, H.A.; Ismail, S.A.; Shafi, M.A.; Al-Ghamdi, A.A. The efficacy of perfusion index as an indicator for intravascular injection of epinephrine-containing epidural test dose in propofol-anesthetized adults. Anesth. Analg. 2009, 108, 549-553. [CrossRef] [PubMed]

32. Korhonen, I.; Yli-Hankala, A. Photoplethysmography and nociception. Acta Anaesthesiol. Scand. 2009, 53, 975-985. [CrossRef] [PubMed]

33. Gigante, A.; Gasperini, M.L.; Rosato, E.; Navarini, L.; Margiotta, D.; Afeltra, A.; Muscaritoli, M. Phase angle could be a marker of microvascular damage in systemic sclerosis. Nutrition 2020, 73, 110730. [CrossRef]

34. Bosy-Westphal, A.; Danielzik, S.; Dörhöfer, R.P.; Later, W.; Wiese, S.; Müller, M.J. Phase angle from bioelectrical impedance analysis: Population reference values by age, sex, and body mass index. JPEN J. Parenter. Enteral Nutr. 2006, 30, 309-316. [CrossRef] [PubMed]

35. Albrecht, D.S.; Ahmed, S.U.; Kettner, N.W.; Borra, R.J.H.; Cohen-Adad, J.; Deng, H.; Houle, T.T.; Opalacz, A.; Roth, S.A.; Melo, M.F.V.; et al. Neuroinflammation of the spinal cord and nerve roots in chronic radicular pain patients. Pain 2018, 159, 968-977. [CrossRef] [PubMed]

36. Barrea, L.; Pugliese, G.; de Alteriis, G.; Colao, A.; Savastano, S.; Muscogiuri, G. Phase Angle: Could Be an Easy Tool to Detect Low-Grade Systemic Inflammation in Adults Affected by Prader-Willi Syndrome? Nutrients 2020, 12, 2065. [CrossRef] [PubMed] 